\title{
The Elastic Energy-Momentum Tensor in Special Relativity
}

\author{
DaVID N. Williams \\ Randall Laboratory of Physics, The University of Michigan, \\ Ann Arbor, Michigan 48109-1120
}

Received June 15, 1989

\begin{abstract}
We consider the standard nonrelativistic theory of a continuous, elastic medium with finite deformations, according to which the elastic energy is a function only of the state of strain, and the elastic stress tensor is proportional to the strain gradient of the elastic energy in appropriate coordinates. We derive a special relativistic, energy-momentum tensor, which yields the standard class of theories in the nonrelativistic limit, from the requirement that it depend only on the state of deformation (including the minimal dependence on velocity consistent with covariance), plus conservation laws. The result agrees with an earlier theory proposed by B. DeWitt (in "Gravitation: An Introduction to Current Research" (L. Witten, Ed.), pp. 305-318, Wiley, New York, 1962), who generalized the nonrelativistic Lagrangian to gencral relativity. The clastic momentum density turns out to be of order $v / c^{2}$, and therefore absent in the nonrelativistic theory. 1989 Academic Press, Inc.
\end{abstract}

\section{INTRODUCTION}

Some early, sketchy remarks on the special relativistic version of the stressenergy-momentum tensor (called the energy-momentum tensor) for a continuous, ideal, elastic medium were given by Pauli [1]. The nonrclativistic theory of elasticity for finite deformations, including the theory of elastic waves, has undergone considerable development since then, which we shall not even begin to trace. A refined mathematical formalism for nonrelativistic elasticity now appears in standard texts [2], which we summarize later, in Section 3.

C. B. Rayner [3] has a discussion of elasticity in general relativity, which includes some references to earlier work. The definitive treatment appears to be the later work of B. DeWitt [4]. We did not know about DeWitt's elegant treatment at the time we did the work in the present paper, having learned of it only recently when we came across an application of his theory to collapsing stars by Gerlach and Scott [6]. DeWitt straightforwardly generalizes the nonrelativistic Lagrangian to general relativity, to lay a foundation for measurements in a larger attempt to quantize gravity. We very much recommend his discussion. ${ }^{1}$

${ }^{1}$ There is a paper by W. C. Davidon [5] on continuum mechanics in special relativity, which does not go into elasticity explicitly. He appears not to have been aware of DeWitt's work. 
Our treatment arrives at the same answer in the more restricted framework of special relativity, by a different method. It is also straightforward, being essentially an invariance argument based on the requirement that the elastic tensor depend only on distortion variables, be covariant, and obey the work-energy conservation law. We present it here in the interest of having an independent treatment, selfcontained within the simpler environment of special relativity. ${ }^{2}$

We also provide the answer to a question which we were unable to find in the nonrelativistic literature: what is the momentum density for an elastic medium? The generalization from the nonrelativistic to the relativistic theory provides the answer to that question automatically: the elastic momentum density is zero in the nonrelativistic theory, because it contains a factor $v / c^{2}$ in the relativistic theory. That seems not inconsistent with what one might expect from a quantum picture. Kittel, for example, gives an argument for zero momentum in all phonons except for the infinite wavelength mode [7].

We begin in Section 2 by reviewing some notation for the Lagrangian coordinate description of continuous media. The nonrelativistic model of elastic media with finite deformations in the version of Green and Zerna [2] is reviewed in Section 3. The conservation law constraints, both relativistic and nonrelativistic, are reviewed in Section 4. In Section 5, we introduce a relativistic description of the state of distortion of a continuous medium (whether elastic or not) by means of a triad of four-vector distortion fields, actually a tetrad when the four-velocity is included. Information about the distortion of aging of body elements due to different proper times is not included. ${ }^{3}$

Then in Section 6 we derive the parametrization of the relativistic version of the elastic tensor in terms of an elastic potential energy function, which depends only on elastic moduli and strain invariants, and in terms of the distortion and four-velocity fields. We show that parametrization to be sufficient to satisfy the relativistic conservation laws, and we make what seems to us a convincing case for the nonexistence of other solutions.

We conclude in Section 7 with a discussion of the nonrelativistic limit, verifying in particular that the elastic stress and energy have the correct limits and that the momentum density vanishes in that limit.

\section{Deformation in Lagrangian CoORdinates}

Our notation is similar to, but not identical with, that of Green and Zerna [2]. We assign the Cartesian coordinates

$$
\alpha=\left(\alpha^{1}, \alpha^{2}, \alpha^{3}\right)
$$

\footnotetext{
${ }^{2}$ We have also completed a companion study of classical, relativistic elastic strings, which may be submitted for publication later.

${ }^{3}$ De Witt [4] had realized this before us.
} 
to the mass elements of the medium in its undeformed, unmoving, reference state. The Cartesian three-vector position of each mass element (unless we state otherwise, "mass element" means "rest mass element") at the time $t$ in an inertial frame is given by the family of motions

$$
\mathbf{x}_{t}(\boldsymbol{\alpha})=\mathbf{x}(t, \boldsymbol{\alpha}),
$$

which we assume to be one-to-one (nonsingular) at fixed $t$ and sufficiently differentiable in $t$ and $\boldsymbol{\alpha}$. Associated with these motions is the three-vector velocity field

$$
\mathbf{v}_{t}(\boldsymbol{\alpha})=\mathbf{v}(t, \boldsymbol{\alpha})=\frac{\partial}{\partial t} \mathbf{x}(t, \boldsymbol{\alpha}),
$$

which represents the velocity at time $t$ of the mass element labeled by $\boldsymbol{\alpha}$. The volume of an undistorted mass element is then $d^{3} \alpha$. Corresponding to any given rest mass density $\rho_{0}(\boldsymbol{\alpha})$ in the reference state, we have the rest mass density at time $t$ :

$$
\begin{aligned}
\rho(t, \mathbf{x}) & =\rho_{0}(\boldsymbol{\alpha}) / J_{t}, \\
J_{t} & \equiv \operatorname{det} \frac{\partial \mathbf{x}_{t}}{\partial \boldsymbol{\alpha}} .
\end{aligned}
$$

The rest mass element is

$$
d m=\rho(t, \mathbf{x}) d^{3} x=\rho_{0}(\boldsymbol{\alpha}) d^{3} \alpha .
$$

The continuity equation follows,

$$
\partial_{\imath} \rho+\nabla \cdot(\rho \mathbf{v})=0
$$

where we use the notation

$$
\hat{\partial}_{t}=\left.\frac{\partial}{\partial t}\right|_{x}, \quad \nabla=\frac{\partial}{\partial \mathbf{x}},
$$

for the partial time derivative holding $\mathbf{x}$ fixed and the usual spatial gradient holding $t$ fixed. We use the notation

$$
D_{t}=\left.\frac{\partial}{\partial t}\right|_{\alpha}=\partial_{t}+\mathbf{v} \cdot \nabla
$$

for the material time derivative, or time derivative at fixed $\alpha$, which is the time derivative appearing in the definition of the velocity field, Eq. (3).

Let $\mathbf{e}_{i}=\mathbf{e}^{i}, i=1,2,3$, be a constant, right-handed system of orthogonal unit three-vectors. We use these as Cartesian basis vectors both in the reference body three-space and in the inertial three-space where the motion occurs. We follow the convention that Latin indices $i, j, \ldots$ refer to inertial space while $s, t, \ldots$ refer to 
reference space components, ${ }^{4}$ with the summation convention for repeated indices. Neighboring points in the undistorted body at rest are separated by

$$
d \boldsymbol{\alpha}=d \alpha^{s} \mathbf{e}_{s}
$$

At time $t$, the image of the separation between neighboring points under the motion is

$$
d \mathbf{x}=\mathbf{x}(t, \boldsymbol{\alpha}+d \boldsymbol{\alpha})-\mathbf{x}(t, \boldsymbol{\alpha})=\frac{\partial x^{i}}{\partial \alpha^{s}} d \alpha^{s} \mathbf{e}_{i}
$$

The Jacobian matrix $\partial x^{i} / \partial \alpha^{s}$ contains all information about the distortion of the unstrained body volume element $d^{3} \alpha$ into its image $d^{3} x$; it permits us to construct any separation $d \mathbf{x}$ of neighboring points of the distorted body from the undistorted separation $d \boldsymbol{\alpha}$.

The body coordinates $\alpha$ can be written at time $t$ in terms of the inertial coordinates $\mathbf{x}$,

$$
\boldsymbol{\alpha}_{i}(\boldsymbol{x})=\boldsymbol{\alpha}(t, \mathbf{x})
$$

where

$$
\boldsymbol{\alpha}\left(t, \mathbf{x}_{t}(\boldsymbol{\alpha})\right)=\boldsymbol{\alpha}
$$

The body coordinates $\boldsymbol{\alpha}_{\boldsymbol{t}}$ are curvilinear with respect to the inertial coordinates $\mathbf{x}$, and the vectors

$$
\boldsymbol{\eta}_{s} \equiv \frac{\partial \mathbf{x}}{\partial \alpha^{s}}, \quad s=1,2,3
$$

point along the direction of increasing $\alpha^{s}$, the other components of $\boldsymbol{\alpha}$ being held fixed, at a given point $\mathbf{x}$. The lengths of these vectors describe the amount of dilation of the reference body along the curvilinear $\alpha^{s}$ axes.

The reciprocal vectors are

$$
\xi^{s}=\nabla \alpha^{s}, \quad \xi^{s} \cdot \boldsymbol{\eta}_{t}=\delta_{i}^{s} .
$$

The elements of length, unconstrained and at time $t$, respectively, are given by

$$
\begin{aligned}
& d \boldsymbol{\alpha} \cdot d \boldsymbol{\alpha}=\delta_{s t} d \alpha^{s} d \alpha^{t}=\nabla_{i} \boldsymbol{\alpha} \cdot \nabla_{j} \boldsymbol{\alpha} d x^{i} d x^{j} \\
& d \mathbf{x} \cdot d \mathbf{x}=\frac{\partial \mathbf{x}}{\partial \alpha^{s}} \cdot \frac{\partial \mathbf{x}}{\partial \alpha^{i}} d \alpha^{s} d \alpha^{t}=\delta_{i j} d x^{i} d x^{j}
\end{aligned}
$$

\footnotetext{
${ }^{4}$ The context should distinguish the cases where the subscript $t$ is used to mean time instead.
} 
The strain tensor is defined by

$$
\begin{gathered}
\frac{1}{2}(d \mathbf{x} \cdot d \mathbf{x}-d \boldsymbol{\alpha} \cdot d \boldsymbol{\alpha})=\gamma_{s t} d \alpha^{s} d \alpha^{t}=\Gamma_{i j} d x^{i} d x^{j}, \\
\gamma_{s t}=\frac{1}{2}\left(\frac{\partial \mathbf{x}}{\partial \alpha^{s}} \cdot \frac{\partial \mathbf{x}}{\partial \alpha^{t}}-\delta_{s t}\right)=\frac{1}{2}\left(\boldsymbol{\eta}_{s} \cdot \boldsymbol{\eta}_{t}-\delta_{s t}\right), \\
\Gamma_{i j}=\frac{1}{2}\left(\delta_{i j}-\nabla_{i} \boldsymbol{\alpha} \cdot \nabla_{j} \boldsymbol{\alpha}\right), \\
\tilde{\Gamma}=\gamma_{s t} s^{s} \xi^{i}=\Gamma_{i j} \mathbf{e}^{i} \mathbf{e}^{j} .
\end{gathered}
$$

Although the strain tensor at a single point does not contain the full information in the Jacobian matrix, it determines the curvilinear metric.

For future reference, we note that the quantity

$$
\lambda^{s t}=\frac{1}{2}\left(\delta^{s t}-\nabla \alpha^{s} \cdot \nabla \alpha^{t}\right)=\frac{1}{2}\left(\delta^{s t}-\xi^{s} \cdot \xi^{t}\right)
$$

contains exactly the same information as $\gamma_{s t}$, because the matrix $\xi^{s} \cdot \xi^{t}$ is the inverse of the matrix $\boldsymbol{\eta}_{s} \cdot \boldsymbol{\eta}_{t}$; and the strain tensor can also be written

$$
\ddot{\Gamma}=\lambda^{s t} \boldsymbol{\eta}_{s} \boldsymbol{\eta}_{l} .
$$

\section{Nonrelativistic Elastic Stress Tensor}

Our convention for the sign of the three-dimensional stress tensor is that

$$
d \mathbf{F}=\vec{S} \cdot d \mathbf{A}
$$

is the force exerted by the medium behind the right-hand oriented surface element $d \mathbf{A}$ on the medium just in front of $d \mathbf{A}$. The front side is the side in the direction of $d \mathbf{A}$. According to the standard theory of an ideal, elastic medium, there are no internal states other than the state of strain, i.e., no thermodynamic variables such as temperature or pressure play a role. All internal stresses are the result of strain. The elastic energy per volume is potential energy, a function only of the state of strain and the body constraints of the form

$$
U(t, \mathbf{x})=F\left(\gamma_{s t}, \boldsymbol{\alpha}\right) / J .
$$

The explicit dependence on $\boldsymbol{\alpha}$ in this expression is a generalized description of the dependence on "elastic moduli," which are allowed to vary from point to point of the body. In particular, we may use the explicit $\alpha$ dependence to define the boundaries of the medium; for example, if there is no medium outside a bounded region of the reference space, we demand that $F$ vanish outside that region. Typically, $F$ might have pieces proportional to various moduli; and the vanishing of $F$ outside the body could then be handled by letting the moduli go smoothly to zero within 
some boundary layer region of arbitrarily small size. But in general, we allow an arbitrary, explicit $\boldsymbol{a}$ dependence.

The potential energy hypothesis in Eq. (20), plus the principle of virtual work, or a standard thermodynamic argument [2], leads to the conclusion that the elastic stress tensor has the form

$$
\begin{aligned}
\vec{S} & =S^{i j} \mathbf{e}_{i} \mathbf{e}_{j}=\sigma^{s t} \boldsymbol{\eta}_{s} \boldsymbol{\eta}_{t}, \\
\sigma^{s t} & =-\frac{1}{J} \frac{\partial F}{\partial \gamma_{s t}}
\end{aligned}
$$

The first of these equations and the fact that the local stress tensor $\sigma^{s t}$ is symmetric are not restricted to elastic media. The second, Eq. (22), is.

The above formalism is broad enough to handle elastically homogeneous or inhomogeneous, isotropic or anisotropic media. The most familiar class of elastic media is that which obeys Hooke's law: stress is proportional to strain.

For use in the relativistic generalization, we note an alternative description of the above theory, namely, we use as a variable the convariant metric tensor,

$$
\eta_{s t} \equiv \boldsymbol{\eta}_{s} \cdot \boldsymbol{\eta}_{t}=\frac{\partial \mathbf{x}}{\partial \alpha^{s}} \cdot \frac{\partial \mathbf{x}}{\partial \alpha^{t}}
$$

instead of the strain tensor $\gamma_{s t}$. We use the same symbol $F=F\left(\eta_{s t}, \boldsymbol{\alpha}\right)$, whereupon

$$
\sigma^{s t}=-\frac{2}{J} \frac{\partial F}{\partial \eta_{s t}} .
$$

For some purposes, it is convenient to use the contravariant metrix tensor

$$
\xi^{s t}=\xi^{s} \cdot \xi^{t}=\nabla \alpha^{s} \cdot \nabla \alpha^{t}
$$

as a variable instead of $\eta_{s t}$, in which case we write

$$
F\left(\eta_{s t}, \boldsymbol{\alpha}\right)=G\left(\xi^{s t}, \boldsymbol{\alpha}\right)
$$

It is straightforward to verify that

$$
S^{i j}=\frac{\partial x^{j}}{\partial \alpha^{s}} \frac{\partial x^{j}}{\partial \alpha^{t}} \sigma^{s t}=\frac{2}{J} \frac{\partial \alpha^{s}}{\partial x^{i}} \frac{\partial \alpha^{t}}{\partial x^{j}} \frac{\partial G}{\partial \xi^{s t}} .
$$

If we use the strain metric as a lowering symbol to define a local stress tensor with lower (covariant) body indices,

$$
\zeta_{s t}=\eta_{s s} \cdot \eta_{t t^{\prime}} \sigma^{s^{\prime} t}
$$


then this can be written

$$
\begin{aligned}
& S_{i j}=\frac{\partial \alpha^{s}}{\partial x^{i}} \frac{\partial \alpha^{t}}{\partial x^{j}} \zeta_{s t}=S^{i j}, \\
& \zeta_{s t}=\frac{2}{J} \frac{\partial G}{\partial \xi^{s t}} .
\end{aligned}
$$

The different names $\zeta$ and $\sigma$ for the covariant and contravariant local stress tensors are intended to avoid confusion with the Cartesian raising and lowering operation, which is trivial both in body space and in inertial space. We actually use only the $\sigma$ 's in this paper.

\section{Convenilons for Matekial Equations of MOTION AND THE WORK-ENERGY THEOREM}

We assume only volume external forces, with no external surface forces, not even on the boundaries of the medium. We assume Newton's law of reaction for all internal stresses across surface elements separating neighboring volume elements.

Our Lorentz metric is $(+,-,-,-)$, with four-vector indices $\mu=0,1,2,3$. Latin indices are three-vector indices, as before. We define $x^{0}=c t$, and $\partial_{\mu}=\partial / \partial x^{\mu}$. For convenience in discussing both nonrelativistic and relativistic versions of the theory, we define the energy-momentum tensor in both nonrelativistic units, $T_{\mathrm{NR}}^{\mu v}$, and relativistic units, $T^{\mu \nu}$ :

$$
\begin{aligned}
& T_{\mathrm{NR}}^{00}=T^{00}=\text { energy } / \text { volume } \\
& T_{\mathrm{NR}}^{i 0}=c T^{i 0}=\text { energy } / \text { area-time }=\text { flux of energy, } \\
& T_{\mathrm{NR}}^{0 j}=T^{0 i} / c=\text { momentum } / \text { volume, } \\
& T_{\mathrm{NR}}^{i j}=T^{i j}=\text { momentum } / \text { area-time = force } / \text { area = flux of momentum. }
\end{aligned}
$$

The external force density four-vector $f^{\mu}$ is

$$
f=(\mathbf{f} \cdot \mathbf{v} / c, \mathbf{f}),
$$

where $\mathrm{f} d^{3} x$ is the external force on a volume element, and $\mathbf{f} \cdot \mathbf{v} d^{3} x$ is the power expended by the external force on the volume element. The equations of motion are

$$
\partial_{\mu} T^{\mu v}=f^{v},
$$

or in nonrelativistic units,

$$
\begin{array}{ll}
\partial_{t} T_{\mathrm{NR}}^{00}+\partial_{i} T_{\mathrm{NR}}^{i 0}=\mathbf{f} \cdot \mathbf{v}, & \text { (power law) } \\
\partial_{t} T_{\mathrm{NR}}^{0 j}+\partial_{i} T_{\mathrm{NR}}^{i j}=f^{j} . & \text { (force law) }
\end{array}
$$


We introduce the covariant four-velocity field $u^{\mu}(x)$ :

$$
u=\gamma(v)(c, \mathbf{v}), \quad u \cdot u=c^{2} .
$$

Then $f \cdot u=0$, and the relativistic conservation law, or, in nonrelativistic units, the work-energy theorem, says

$$
u_{v} \partial_{\mu} T^{\mu v}=0
$$

We decompose $T^{\mu \nu}$ into a kinetic part and the rest:

$$
T^{\mu v}=K^{\mu v}+E^{\mu v}
$$

The kinetic part in the nonrelativistic case is

$$
\begin{array}{ll}
K_{\mathrm{NR}}^{00}=\frac{1}{2} \rho v^{2}, & K_{\mathrm{NR}}^{0 j}=\rho v^{j}, \\
K_{\mathrm{NR}}^{i 0}=v^{i} K_{\mathrm{NR}}^{00}, & K_{\mathrm{NR}}^{i j}=v^{i} K_{\mathrm{NR}}^{0 j} .
\end{array}
$$

In the relativistic case it is

$$
K^{\mu v}=\frac{\rho}{\gamma} u^{\mu} u^{v}
$$

In both cases, flux is material flow.

Both kinetic tensors automatically obey the conservation law (36) because of material identities, such as the continuity equation. Thus,

$$
u_{v} \partial_{\mu} E^{\mu v}=0 .
$$

We are interested in the situation where $E^{\mu v}$ describes the elastic properties of the medium. The procedure will be to make a general Ansatz for $E^{\mu \nu}$ and look for conditions for the conservation law to be valid. We then check that we recover the class of theories described in Section 3 in the nonrelativistic limit, in effect rederiving the fact that elastic stress is the gradient of strain in the nonrelativistic theory; and we check that the physical properties in the relativistic regime are natural for an elastic tensor that one would be willing to call the relativistic generalization of the nonrelativistic theory.

\section{The Covariant Distortion Fields}

As we reviewed in Section 2 , the vectors $\eta_{s}$ have an intuively simple relation to the state of distortion or strain (we use those terms interchangeably). For the relativistic generalization, we find it convenient to start from the reciprocal 
quantities $\xi^{s}=\nabla \alpha^{s}$ as a complete description of distortion. The reason is that not only do the quantities

$$
\xi_{\mu}^{s}(x)=\partial_{\mu} \alpha^{s}(t, \mathbf{x}), \quad s=1,2,3,
$$

transform as four-vector fields under Lorentz transformations, they have somewhat simpler algebraic properties then their reciprocals, while containing the same distortion information. We call this set of three, four-vector distortion fields a distortion triad.

They can be put together with the local four-velocity field,

$$
u(x)=\gamma D_{\imath} x \equiv D_{\tau} x,
$$

to form a tetrad. Since

$$
D_{t} \alpha^{s}=0
$$

we get

$$
\partial_{0} \alpha^{s}=-\frac{\mathbf{v}}{c} \cdot \nabla \alpha^{s}
$$

which in turn yields

$$
u \cdot \xi^{s}=0
$$

Hence $\xi^{s}$ is spacelike. Moreover, the tetrad $\left\{u, \xi^{s}\right\}$ is linearly independent, because the determinant

$$
\varepsilon^{\mu \nu \lambda \rho} u_{\mu} \xi_{v}^{1} \xi_{\lambda}^{2} \xi_{\rho}^{3}=\frac{c}{J \gamma}, \quad \varepsilon_{0123} \equiv-1,
$$

is nonvanishing. This incidentally shows that $J \gamma$ is a Lorentz invariant.

It turns our that the tetrad $\left\{u, \eta_{s}\right\}$ reciprocal to $\left\{u, \xi^{s}\right\}$ is convenient for applications, although algebraically a little more complicated. It obeys

$$
\eta_{s} \cdot \xi^{t}=\delta_{s}^{t}, \quad \eta_{s} \cdot u=0,
$$

To describe it and help in keeping track of what variables are held fixed in partial derivatives, we introduce the following notation for the world position four-vector:

$$
y^{\mu}(\tau, \boldsymbol{\alpha})=x^{\mu}(t, \boldsymbol{\alpha})=\left(c t, \mathbf{x}_{t}(\boldsymbol{\alpha})\right),
$$

where $\tau(t, \alpha)$ is the proper time of the body point $\alpha$ at time $t$,

$$
\tau(t, \boldsymbol{\alpha})=\tau\left(t_{0}, \boldsymbol{\alpha}\right)+\int_{t_{0}}^{t} \frac{d t^{\prime}}{\gamma\left(t^{\prime}, \boldsymbol{\alpha}\right)} .
$$


In this notation,

$$
u=\frac{\partial y}{\partial \tau}=\gamma \frac{\partial x}{\partial \iota}
$$

and is straightforward to verify that Eq. (47) is solved by

$$
\eta_{s}^{\mu}=\frac{\partial y^{\mu}}{\partial \alpha^{s}}-\frac{u^{\mu}}{c} \frac{u}{c} \cdot \frac{\partial y}{\partial \alpha^{s}}=\frac{\partial x^{\mu}}{\partial \alpha^{s}}-\frac{u^{\mu}}{c} \frac{u}{c} \cdot \frac{\partial x}{\partial \alpha^{s}}
$$

The notation using $y$ and $x$ indicates that $\tau$ and $t$, respectively, are held fixed in the differentiation with respect to $\boldsymbol{\alpha}$.

The information that is being omitted from $\eta_{s}$ by projecting the $u$ direction out of the triad of four-vectors $\partial y / \partial \alpha^{s}$ can be understood as follows. Note that

$$
\begin{aligned}
\left.\frac{\partial \tau(t, \boldsymbol{\alpha})}{\partial \alpha^{s}}\right|_{\tau}=0 & =\left.\left.\frac{\partial \tau}{\partial t}\right|_{\alpha} \frac{\partial t}{\partial \alpha^{s}}\right|_{\tau}+\left.\frac{\partial \tau}{\partial \alpha^{s}}\right|_{t} \\
& =\left.\frac{1}{\gamma} \frac{\partial t}{\partial \alpha^{s}}\right|_{\tau}+\left.\frac{\partial \tau}{\partial \alpha^{s}}\right|_{t}
\end{aligned}
$$

Therefore,

$$
\frac{\partial y}{\partial \alpha^{s}}=\frac{\partial x}{\partial \alpha^{s}}+\left.\frac{\partial x}{\partial t} \frac{\partial t}{\partial \alpha^{s}}\right|_{\tau}=\frac{\partial x}{\partial \alpha^{s}}-\left.u \frac{\partial \tau}{\partial \alpha^{s}}\right|_{t},
$$

so

$$
\frac{\partial y^{0}}{\partial \alpha^{s}}=-\left.\gamma c \frac{\partial \tau}{\partial \alpha^{s}}\right|_{t}, \quad \frac{\partial \mathbf{y}}{\partial \alpha^{s}}=\frac{\partial \mathbf{x}}{\partial \alpha^{s}}-\left.\gamma \mathbf{v} \frac{\partial \tau}{\partial \alpha^{s}}\right|_{t},
$$

whereas

$$
\eta_{s}^{0}=\gamma^{2} \frac{\mathbf{v}}{c} \cdot \frac{\partial \mathbf{x}}{\partial \alpha^{s}}, \quad \boldsymbol{\eta}_{s}=\frac{\partial \mathbf{x}}{\partial \alpha^{s}}-\gamma^{2} \frac{\mathbf{v}}{c} \frac{\mathbf{v}}{c} \cdot \frac{\partial \mathbf{x}}{\partial \alpha^{s}}
$$

The information that is missing from $\eta_{s}$ is clearly $\partial \tau /\left.\partial \alpha^{s}\right|_{t}$, which describes the relative aging of different body points.

We take as the relativistic analog of the strain tensor, or rather of the strain metric $\eta_{s} \cdot \eta_{l}$, the symmetric, $3 \times 3$ distortion array of Lorentz invariants:

$$
\eta_{s t} \equiv-\eta_{s} \cdot \eta_{t}=-\frac{\partial y}{\partial \alpha^{s}} \cdot \frac{\partial y}{\partial \alpha^{t}}+\frac{u}{c} \cdot \frac{\partial y}{\partial \alpha^{s}} \frac{u}{c} \cdot \frac{\partial y}{\partial \alpha^{t}} .
$$

The reciprocal, invariant metric tensor is

$$
\xi^{s t} \equiv-\xi^{s} \cdot \xi^{t}=\mathbf{V} \alpha^{s} \cdot \mathbf{V} \alpha^{t}-\frac{\mathbf{v}}{c} \cdot \mathbf{V} \alpha^{s} \frac{\mathbf{v}}{c} \cdot \mathbf{V} \alpha^{\prime}
$$


We could define the Lorentz invariant "analogous" strain tensor (see Eq. (16)),

$$
\gamma_{s t}=-\frac{1}{2}\left(\delta_{s t}-\eta_{s t}\right),
$$

or the Lorentz invariant "alternative" strain tensor (see Eq. (17)),

$$
\lambda_{s t}=\frac{1}{2}\left(\delta_{s t}-\left(\eta^{-1}\right)_{s t}\right) .
$$

We choose instead to describe the state of strain by the invariant metric tensor $\eta_{s t}$. All of the above quantities agree with their nonrelativistic analogs to leading order in $v / c$.

Finally, we note an identity which shows the Lorentz invariant $J \gamma$ to be a function only of the relativistic strain:

$$
\begin{gathered}
\varepsilon^{\mu}{ }_{v \lambda \rho} \eta_{1}^{v} \eta_{2}^{\lambda} \eta_{3}^{\rho}=-J \gamma \frac{u^{\mu}}{c}, \\
\operatorname{det}\left(-\eta_{s} \cdot \eta_{t}\right)=(J \gamma)^{2} .
\end{gathered}
$$

The corresponding identity for the reciprocal quantities is

$$
\begin{aligned}
\varepsilon^{\mu v \rho} \xi_{\nu}^{1} \xi_{\lambda}^{2} \xi_{\rho}^{3} & =\frac{1}{J \gamma} \frac{u^{\mu}}{c}, \\
\operatorname{det}\left(-\xi^{s} \cdot \xi^{t}\right) & =\frac{1}{(J \gamma)^{2}} .
\end{aligned}
$$

\section{The Relativistic Elastic Tensor}

In order to motivate our Ansatz for the elastic tensor, let us elaborate a bit on the concept of elasticity. In its nonrelativistic conception, the stress in an elastic medium depends only on the field of elastic moduli and the state of distortion at each point, except for the flow term in the kinetic part of the tensor; and the energy density, aside from the kinetic part, is also a function only of the clastic moduli and the state of distortion. In the relativistic generalization, we allow the elastic part $E^{\mu v}$ of the energy-momentum tensor to depend only on the state of distortion, generalized to covariant form, the four-velocity field, to account for relativistic kinematic effects, and the elastic moduli of the body. In other words, $E^{\mu v}$ is to depend only on the elastic moduli, which for us means any explicit $\boldsymbol{\alpha}$ dependence, and on the four-velocity-four-distortion tetrad.

Because the tetrad $\left\{u, \eta_{s}\right\}$ is linearly independent, any second rank tensor can be decomposed into the 16 dyads formed from it,

$$
E^{\mu v}=\frac{u^{\mu} u^{v}}{c^{2}} \sigma^{00}+\eta_{s}^{\mu} \eta_{t}^{v} \sigma^{s t}+\frac{u^{\mu}}{c} \eta_{r}^{v} \sigma^{0 \imath}+\eta_{s}^{\mu} \frac{u^{v}}{c} \sigma^{s 0},
$$


where we use the summation convention for the body indices $s, t=1,2,3$. The $\sigma$ coefficients must be Lorentz invariant fields (in fact, Poincare invariant), because we want $E^{\mu v}$ to be covariant. They can have an explicit $\alpha$ dependence, because the body coordinates are Poincare invariant. If we demand that $E^{\mu v}$ depend only on distortion, velocity, and moduli, the only other dependence the $\sigma$ 's can have is on invariants formed from the tetrad $\left\{u, \eta_{s}\right\}$. We saw earlier that all scalar and pseudoscalar invariants formed from these depend only on the strain invariants $\eta_{s t}=-\eta_{s} \cdot \eta_{t}$. Thus, we may choose to write the most general parametrization of $\sigma^{00}$, for example, in the form

$$
\sigma^{00}=\frac{1}{J \gamma} F\left(\eta_{s t}, \boldsymbol{\alpha}\right),
$$

where we are using the fact that $J y$ is a function of $\eta_{s t}$.

The only other general constraint is the conservation law, which says

$$
\begin{aligned}
0= & u_{v} \partial_{\mu} E^{\mu v} \\
= & \frac{1}{J \gamma} u \cdot \partial\left(J \gamma \sigma^{00}\right)+u_{v}\left(\eta_{s} \cdot \partial \eta_{t}^{v}\right) \sigma^{s t} \\
& +\frac{u_{v}}{J \gamma c} u \cdot \partial\left(\eta_{s}^{v} \cdot J \gamma \sigma^{0 s}\right)+c\left(\partial \cdot \frac{\eta_{s}}{J \gamma}\right) J \gamma \sigma^{s 0}+\frac{u_{v}}{J \gamma c} \eta_{s} \cdot \partial\left(u^{v} J \gamma \sigma^{s 0}\right),
\end{aligned}
$$

where we have used the orthogonality of $u$ with $\eta_{s}$ and the continuity equation

$$
\partial \cdot \frac{u}{J \gamma}=0
$$

which follows from Eqs. (6) and (4) by putting $\rho_{0}=1$.

To analyze this, we note some identities among partial derivatives. First the continuity equation (67) may be regarded as a straightforward property of the Jacobian matrix of the transformation between the variables $y$ (or $x)$ and $(\tau, \boldsymbol{\alpha})$, which also entails that

$$
\partial \cdot\left(\frac{1}{J \gamma} \frac{\partial y}{\partial \alpha^{s}}\right)=0
$$

This leads to

$$
\partial \cdot \frac{\eta_{s}}{J \gamma}=-\frac{a \cdot \eta^{s}}{J \gamma c^{2}}=-\frac{\dot{\kappa}_{s}}{J \gamma c},
$$

where we have introduced the four-acceleration

$$
a \equiv \dot{u} \equiv D_{\imath} u, \quad a \cdot u=0,
$$


and the notation

$$
\kappa_{s} \equiv \frac{u}{c} \cdot \frac{\partial y}{\partial \alpha^{s}}, \quad \dot{\kappa}_{s} \equiv D_{\tau} \kappa_{s}=\frac{a}{c} \cdot \eta_{s}=-\frac{u}{c} \cdot \dot{\eta}_{s} .
$$

Note that

$$
\eta_{s}=\frac{\partial y}{\partial \alpha^{s}}-\frac{u}{c} \kappa_{s}
$$

and that the invariants $\kappa_{s}$ contain the relative aging information that is absent in $\eta_{s}$.

We also define the invariant derivatives

$$
D_{s} \equiv \eta_{s} \cdot \partial=\frac{\partial}{\partial \alpha^{s}}-\frac{\kappa_{s}}{c} \frac{\partial}{\partial \tau},
$$

and record the symmetric and antisymmetric identities

$$
\begin{aligned}
u_{\mu}\left(D_{s} \eta_{t}^{\mu}+D_{t} \eta_{s}^{\mu}\right) & =\kappa_{s} \dot{\kappa}_{t}+\kappa_{t} \dot{\kappa}_{s}-\frac{\partial u}{\partial \alpha^{s}} \cdot \eta_{t}-\frac{\partial u}{\partial \alpha^{t}} \cdot \eta_{s} \\
& =-\frac{\partial}{\partial \tau}\left(\eta_{s} \cdot \eta_{t}\right)=\dot{\eta}_{s t}, \\
u_{\mu}\left(D_{s} \eta_{t}^{\mu}-D_{t} \eta_{s}^{\mu}\right) & =\kappa_{s} \dot{\kappa}_{t}-\kappa_{t} \dot{\kappa}_{s}-\frac{\partial u}{\partial \alpha^{s}} \cdot \eta_{t}+\frac{\partial u}{\partial \alpha^{l}} \cdot \eta_{s} .
\end{aligned}
$$

The strategy now is to identify kinematic and deformation quantities in the conservation law (66) which vary independently in an arbitrary motion and which must have coefficients that vanish separately if the elastic tensor is to be defined independently of the equations of motion. Keeping in mind that the $\sigma$ 's have no explicit $\tau$ dependence and the identities above, the conservation law becomes

$$
\begin{aligned}
0= & \dot{\eta}_{s t}\left(\frac{1}{J \gamma} \frac{\partial\left(J \gamma \sigma^{00}\right)}{\partial \eta_{s t}}+\frac{\sigma^{s t}}{2}\right) \\
& +u \cdot \frac{D_{s} \eta_{t}-D_{t} \eta_{s}}{2} \sigma^{s t} \\
& -\dot{\kappa}_{s}\left(\sigma^{0 s}+\sigma^{s 0}\right)+\frac{c}{J \gamma} \frac{\partial\left(J \gamma \sigma^{s 0}\right)}{\partial \alpha^{s}}-\frac{\kappa_{s} \dot{\eta}_{t u}}{J \gamma} \frac{\partial\left(J \gamma \sigma^{s 0}\right)}{\partial \eta_{t u}}
\end{aligned}
$$

If the kinematic quantities $\dot{\eta}_{s t}$ (symmetric), $u \cdot\left(D_{s} \eta_{t}-D_{t} \eta_{s}\right)$ (antisymmetric), $\dot{\kappa}_{s}$, and $\kappa_{s}$ can be varied independently by selecting an arbitrary motion subject to fixed strain invariants $\eta_{s t}$, we conclude that the $\sigma$ 's must obey the following identities in order to define an elastic tensor independent of equations of motion: 


$$
\begin{aligned}
\sigma^{0 s} & =-\sigma^{s 0}, \quad \frac{\partial\left(J \gamma \sigma^{s 0}\right)}{\partial \alpha^{s}}=0, \quad \frac{\partial\left(J \gamma \sigma^{s 0}\right)}{\partial \eta_{t u}}=0 ; \\
\sigma^{s t} & =\sigma^{t s} ; \\
\sigma^{s t} & =-\frac{2}{J \gamma} \frac{\partial\left(J \gamma \sigma^{00}\right)}{\partial \eta_{s t}} .
\end{aligned}
$$

It is not hard to show that there are indeed nonzero solutions to Eq. (77) for $\sigma^{s 0}$ and $\sigma^{0 s}$, but that their contributions to $E^{\mu v}$ all have identically vanishing fourdivergence (zero force density) and may be discarded. The solutions to Eq. (79) are automatically symmetric, satisfying Eq. (78).

We conclude that the class of elastic energy-momentum tensors may be parametrized in the form

$$
\begin{aligned}
E^{\mu \nu} & =\frac{u^{\mu} u^{v}}{c^{2}} \frac{F}{J \gamma}-\eta_{s}^{\mu} \eta_{t}^{v} \frac{2}{J \gamma} \frac{\partial F}{\partial \eta_{s t}}, \\
F & =F\left(\eta_{s t}, \boldsymbol{\alpha}\right) .
\end{aligned}
$$

There is no loss of generality in this parametrization if our independent variation argument following Eq. (76) is accepted. In any case, the parametrization satisfies the general conservation law and the general criteria for elasticity. We shall see by inspection in the next section that its nonrelativistic limit is consistent with the standard theory.

\section{INTERPRETATION AND NONRELATIVISTIC LIMIT}

To interpret the elastic solutions (80), we write out the surviving, symmetric components of the relativistic tensor $E^{\mu \nu}$, Eq. (64), in nonrelativistic units:

$$
\begin{aligned}
& E_{\mathrm{NR}}^{00}=\gamma^{2} \sigma^{00}+\frac{\gamma^{4}}{c^{2}} \mathbf{v} \cdot \frac{\partial \mathbf{x}}{\partial \alpha^{s}} \mathbf{v} \cdot \frac{\partial \mathbf{x}}{\partial \alpha^{t}} \sigma^{s t}, \\
& E_{\mathrm{NR}}^{i 0}=\gamma^{2} v^{i} \sigma^{00}+\left(\gamma^{2} \frac{\partial x^{i}}{\partial \alpha^{s}}-\frac{\gamma^{4}}{c^{2}} v^{i} \mathbf{v} \cdot \frac{\partial \mathbf{x}}{\partial \alpha^{s}}\right) \mathbf{v} \cdot \frac{\partial \mathbf{x}}{\partial \alpha^{t}} \sigma^{s t}, \\
& E_{\mathrm{NR}}^{0 j}=\frac{\gamma^{2}}{c^{2}} v^{j} \sigma^{00}+\frac{\gamma^{2}}{c^{2}} \mathbf{v} \cdot \frac{\partial \mathbf{x}}{\partial \alpha^{s}} \frac{\partial x^{j}}{\partial \alpha^{i}} \sigma^{s t}-\frac{\gamma^{4}}{c^{4}} \mathbf{v} \cdot \frac{\partial \mathbf{x}}{\partial \alpha^{s}} \mathbf{v} \cdot \frac{\partial \mathbf{x}}{\partial \alpha^{t}} v^{j} \sigma^{s t}, \\
& E_{\mathrm{NR}}^{i j}=\frac{\gamma^{2}}{c^{2}} v^{i} v^{j} \sigma^{00}+\left(\frac{\partial x^{i}}{\partial \alpha^{s}}-\frac{\gamma^{2}}{c^{2}} v^{i} \mathbf{v} \cdot \frac{\partial \mathbf{x}}{\partial \alpha^{s}}\right)\left(\frac{\partial x^{j}}{\partial \alpha^{t}}-\frac{\gamma^{2}}{c^{2}} v^{j} \mathbf{v} \cdot \frac{\partial \mathbf{x}}{\partial \alpha^{t}}\right) \sigma^{s t} .
\end{aligned}
$$

By inspection of their explicit $c$-dependence, we conclude that if $E_{\mathrm{NR}}^{\mathrm{on}}$ and $E_{\mathrm{NR}}^{i j}$ are to have finite nonrelativistic limits, then so must $\sigma^{00}$ and $\sigma^{s t}$, where the limit in the $\sigma$ 's is taken by using the limiting values $\eta_{s t}=\boldsymbol{\eta}_{s} \cdot \boldsymbol{\eta}_{t}$ defined in Eqs. (13) and (23) as 
$v / c \rightarrow 0$, and by removing any remaining $c$ dependence through $c \rightarrow \infty$. That gives the nonrelativistic limit

$$
\begin{array}{ll}
E_{\mathrm{NR}}^{00}=\sigma^{00}, & E_{\mathrm{NR}}^{0 j}=0, \\
E_{\mathrm{NR}}^{i 0}=v^{i} \sigma^{\mathrm{m}}+E_{\mathrm{NR}}^{i j} v_{j}, & E_{\mathrm{NR}}^{i j}=\frac{\partial x^{i}}{\partial \alpha^{s}} \frac{\partial x^{j}}{\partial \alpha^{i}} \sigma^{s t},
\end{array}
$$

where the limits in the $\sigma$ 's are understood.

In the nonrelativistic limit, the stress part $E_{N R}^{i j}$, parametrized by Eqs. (79) and (65), agrees as it should with the standard class of stress tensors for elastic media, as expressed by Eqs. (24) and (20). The momentum density is zero, as we claimed. The energy density agrees with the standard form of the elastic potential energy. The flux of energy $E_{\mathrm{NR}}^{i 0}$ has a part due to elastic potential energy and a part due to work done by the elastic stress.

We remark that it is straightforward to verify that the nonrelativistic limit obeys the work-energy theorem exactly. We would be surprised if the analog of the discussion in the preceding section could not be carried through for the nonrelativistic case from the beginning, leading to the same result we got in the limit. The requirement that $\sigma^{00}$ and $\sigma^{s t}$ depend only on Poincare invariants would be replaced by the statement that they can depend only on Gallilei invariants, those Gallilei invariants which involve only distortion being exhausted by the components of the nonrelativistic metric tensor $\eta_{s t}$. In particular, we should then get a direct proof that the nonrelativistic, elastic momentum density is zero.

With the nonrelativistic limit as a guide to the intuition, we can see that the relativistic tensor in Eq. (81) has the form one would expect. ${ }^{5}$ We identify the terms in the relativistic tensor (in nonrelativistic units) as follows. In $E_{\mathrm{NR}}^{00}$, the piece $\gamma^{2} \sigma^{00}$ comes from the contribution

$$
\rho_{\mathrm{el}}=\frac{\gamma \sigma^{00}}{c^{2}}=\frac{F}{J c^{2}}
$$

to the rest mass density from the elastic potential energy. The remaining piece is a relativistic effect in the presence of stress.

The energy flux $E_{\mathrm{NR}}^{i 0}$ has a term due to flow of elastic rest mass and a term due to work done by elastic stress.

The momentum density $E_{\mathrm{NR}}^{0 j}$ has a term $\rho_{\mathrm{el}} \gamma v^{j}$ from the elastic rest mass density, and an additional relativistic term from the elastic stress, analogous to the second piece of the energy density.

The flux of momentum term $E_{\mathrm{NR}}^{i j}$ has a piece due to flow of momentum density $\rho_{\mathrm{e} 1} \gamma v^{j}$ and an elastic stress term.

\footnotetext{
${ }^{5}$ See, for example, Bergmann's dicussion [8] of the form taken by a stress tensor in an inertial frame, given its expression in the local rest frame of a material point.
} 
That concludes our study of the elastic tensor in special relativity. We are in complete agreement with DeWitt [4], and we refer to his work for a discussion of certain topics we have not mentioned, such as the propagation of small disturbances.

\section{ACKNOWLEDGMENTS}

It is a pleasure to thank G. W. Ford for suggestions on the basic literature, and M. Sanders and L. Sander for instructive conversations about elasticity, in Summer 1972, when most of this work was done.

\section{REFERENCES}

1. W. Pauli, "Theory of Relativity," Sections 42, 45, Pergamon, New York, 1958 (transl. by G. Field of Relativitätstheorie, in "Encyklopädie der mathematischen Wissenschaften," Vol. 19, Teubner, Leipzig, 1926).

2. A. E. Green and W. Zerna, "Theoretical Elasticity," Oxford Univ. Press (Clarendon), Oxford, 1954.

3. C. B. Rayner, Elasticity in general relativity, Proc. Roy. Soc. A 272 (1963), 44-53.

4. B. S. DeWIrT, The quantization of geometry, in "Gravitation: An Introduction to Current Research" (L. Witten, Ed.), pp. 305-318, Wiley, New York, 1962.

5. W. C. Davidon, A Lorentz invariant continuum mechanics, Ark. Fys. Sem. Trondheim 11 (1976).

6. U. H. GERLACH AND J. F. SCOTT, Metric elasticity in a collapsing star: Gravitational radiation coupled to torsional motion, Phys. Rev. D 34 (1986), 638-649.

7. C. Kittel, "Introduction to Solid State Physics," pp. 92-94, Wiley, New York, 1986.

8. P. G. Bergmann, "Introduction to the Theory of Relativity," Prentice-Hall, New York, 1942. 